\title{
A New Method for Electrical Discharge Machining of Non-Conductive Engineering Ceramics
}

\author{
Li Xiaopeng ${ }^{1, a}$, Liu Yonghong ${ }^{1, b}$ and Ji Renjie ${ }^{1, c}$ \\ ${ }^{1}$ China University of Petroleum, China \\ aupclxp@gmail.com, bliuyh@upc.edu.cn, cjirenjie202@qq.com
}

Keywords: Electrical discharge grinding; Non-conductive engineering ceramics; Assisting electrode

\begin{abstract}
A novel technique named double electrodes synchronous servo electrical discharge grinding (DESSEDG) for non-conductive engineering ceramics, which integrates the advantages of electrical discharge machining (EDM) and mechanical grinding, is presented to achieve high efficiency machining. DESSEDG Processing equipment using this method was produced. The theory and characteristics are introduced. Processing sample has been machined.
\end{abstract}

\section{Introduction}

Engineering ceramics have excellent properties such as high hardness, low density, oxidation resistance, thermal resistance, wear resistance and so on, and are increasingly widely used in various industry fields. But their high hardness and brittleness make engineering ceramic materials very difficult to machine. ${ }^{[1]}$ The current processing methods generally have the problems of high costs, low efficiency, poor quality, etc., and can't meet the demands of the production.

The engineering ceramics with high conductivity (the resistivity less than $100 \Omega \cdot \mathrm{cm}$ ) ${ }^{[2]}$ can be machined by electrical discharge machining (EDM). But non-conductive engineering ceramics cannot be machined directly by EDM method due to high resistivity.

A novel technique named double electrodes synchronous servo electrical discharge grinding (DESSEDG) for non-conductive engineering ceramics, which integrates the advantages of electrical discharge machining (EDM) and grinding, is presented to achieve high efficiency precision machining. This method employs a conductive grinding wheel which rotates fast on the surface of workpiece, and a sheet electrode which is automatically fed to the place where the wheel and workpiece meet. Discharges happen between wheel and sheet electrode under pulse power supply. The sheet electrode is very thin, so it can be considered that discharges are just on the surface of workpiece. The high temperature and pressure of plasma channel can be directly exerted to the workpiece surface to remove material. Then the sparks eroded layer is machined a second time by grinding of the conductive wheel. The advantages of DESSEDG are high efficiency, low processing costs, environmental pollution-free, and so on.

\section{Principle of DESSEDG}

The equipment of DESSEDG includes a pulse power supply, a servo control system, the working fluid circulation system and the machine body. Machine body includes a controlled two-dimensional movement table, a controlled grinding head which can move up and down along the column, the sheet electrode servomechanism driven by a DC servo motor and the machine bed carrying machine parts. EDM pulse power supply provides the discharge energy required. Control system which controls the table, grinding wheels and sheet electrode according to a predetermined trajectory and speed of movement, so that stable electrical discharge grinds process to proceed continuously to obtain the required machining accuracy and surface quality. The working fluid circulation system provides non-conductive machining emulsion to discharging gap, filters and recycles the emulsion. The process is shown in Fig. 1. 


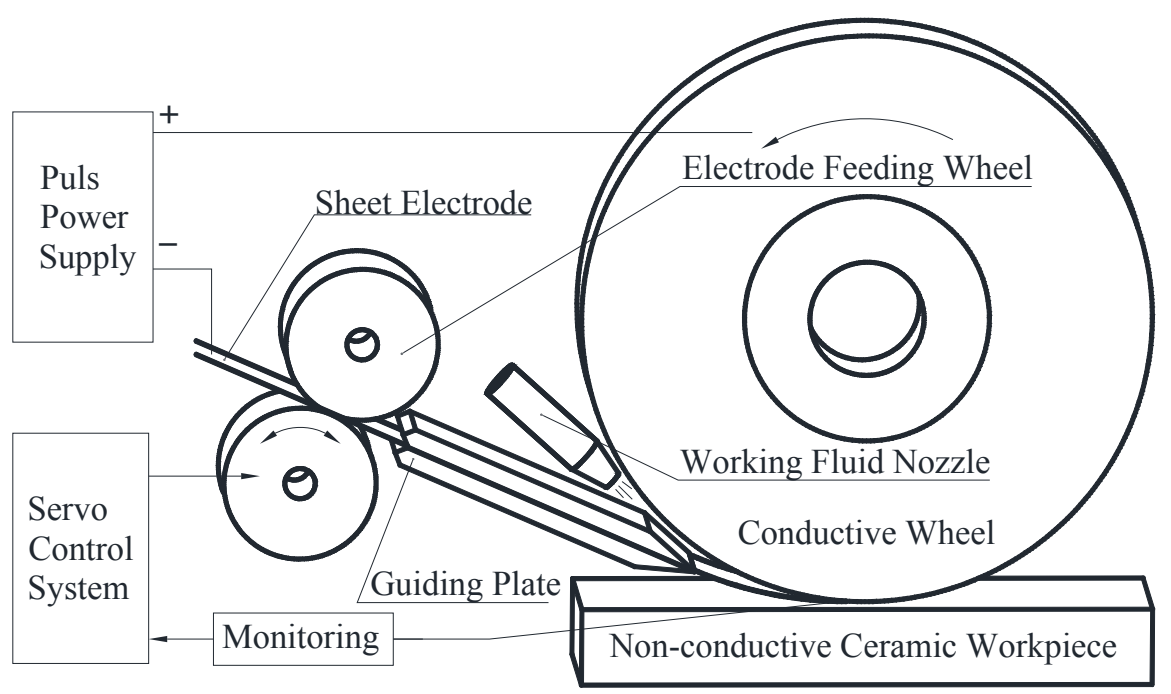

Fig. 1 Schematic of Non-conducting engineering ceramics DESSEDG

In non-conducting engineering ceramics DESSEDG processing, the positive and negative poles of pulse power suply link are conected to conductive grinding wheel and sheet elctrode respectivly. The conductive grinding wheel roating with the spindle at very high speed. Sheet electrode (which is made of copper) feeds along the surface of non-conductive ceramic workpiece automatically driven by DC servo motor. Sheet electrode is pressed close to the surface of non-conducting engineering ceramics by its own flecibility and the pressure of working fluid. When the end of sheet elctrode and the conductive wheel are close enough and the electric field strength reachs ro breakdown strength of the discharge medium, the sparks discharge between the poles. As sheet electrode is very thin (about $0.1 \mathrm{~mm}$ ) and is very close to the surface of the workpiece, so it can be considered that the spark discharging energy directly effects on the non-conducting engineering ceramic surface. Electrical sparks produces instantaneous high temperature (temperature of the center electrical dischargeing channel can be up to $10000{ }^{\circ} \mathrm{C}$ and above) ${ }^{[3]}$ and high pressure (high-temperature thermal expansion of the channel formed by the initial pressure of up to hundreds MPa) ${ }^{[4]}$. Non-conducting engineering ceramics surface instantly melt, gasify and decompose by high-temperature. High temperature and pressure of the discharge channel and the rapid expansion of gasification air bubbles formed in the non-conducting engineering ceramics surface to produce a strong shock wave, causing spalling of the solid phase which doesn't melt. In the discharge process, there're a series derived phenomena, including thermal effects, electromagnetic effects, light effects, sound effects and a wide frequency range of electromagnetic radiation and blast wave, all these can further strengthen erosion of the matierial. Discharge erosion material is thrown out from the machining gap by scraping of high-speed rotating grinding wheel, air absorption, discharge explosive, and high-pressure working fluid impulse injection. When the end of a pulse discharge, it entered deionization pulse interval, for the next pulse discharge preparation. When spark discharge erodes the workpiece, it also make the workpiece to form a metamorphic layer. The metamorphic layer can be removed by scraping of the conductive grinding wheel. So DESSEDG is a composite processing method of electrical discharge machining and mechanical grinding.

\section{Sheet Electrode Feeding Analysis}

Assuming copper sheet electrode in an idealized state that its thickness is zero, its width is just equal to the wheel width, and its sides are precisely aligned with the edge of the wheel, then the sheet electrode can be sent to the location where the wheel surface and the workpiece surface are tangent, making the grinding area and the discharge location is always coincide. In actual processing, the thickness of the copper sheet electrode can't be zero, the discharge will not occur just below the wheel, but occurs in a some distance from the conducting wheel and the non-conductive engineering ceramics contact area, the distance and its control has a greater impact to spark erosion characteristics. 
The larger sheet electrode thickness is, the smaller energy can be conducted to the workpiece surface. Fig. 2 shows the front end of the sheet electrode and the grinding zone.

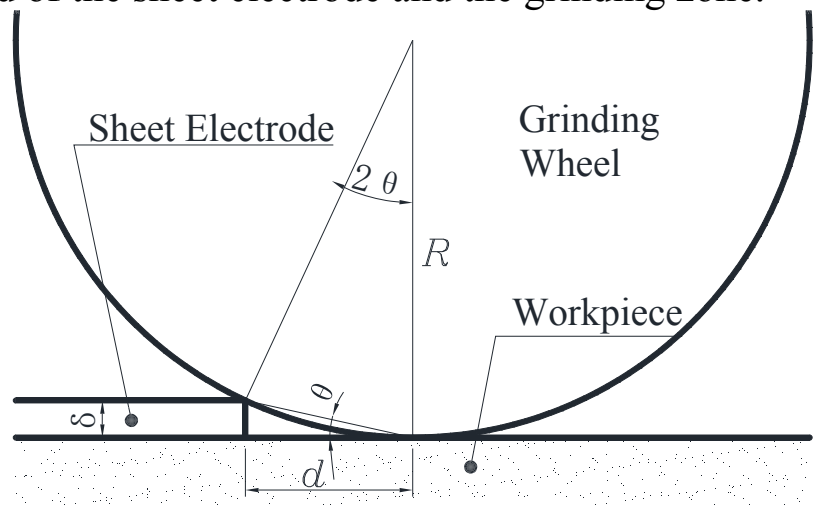

Fig. 2 Area of the front end of sheet electrode and the grinding zone

To simplify the calculation, assuming the sheet electrode being in contact with the wheel (the discharge gap is 0 ), the following formula can be obtained from Fig. 2

$$
\left\{\begin{array}{l}
\tan \theta=\frac{\delta}{d} \\
\sin 2 \theta=\frac{d}{R}=\frac{2 \tan \theta}{1+\tan ^{2} \theta}=\frac{\frac{2 \delta}{d}}{1+\frac{\delta^{2}}{d^{2}}},
\end{array}\right.
$$

The solution is

$$
d=\sqrt{2 \delta R-\delta^{2}}
$$

If the thickness of sheet electrode $\delta=0.15 \mathrm{~mm}$, the wheel Radius $\mathrm{R}=75 \mathrm{~mm}$, inserting them into equation (2),

$$
d=\sqrt{2 \delta R-\delta^{2}}=\sqrt{2 \times 0.15 \times 75-0.15^{2}}=4.71(\mathrm{~mm})
$$

From equation (2), the thinner sheet electrode, the smaller the wheel diameter, the discharge location and the distance between the grinding zone will be the closer, the better discharge erosion can be get. Therefore, in order to enhance the effect of spark discharge erosion, if the rigidity and stability of sheet electrode permission, the thickness of sheet electrode should try to reduce. Copper sheet electrode is driven by the DC servo motor, when the discharge gap is too small or short circuit, the sheet electrode rolls back; when the discharge gap is too large or open circuit, the sheet electrode will quickly be sent to the wheel, so as to maintain the stability of the discharge.

\section{Advantages of DESSEDG}

Non-conducting engineering ceramics DESSEDG method has many advantages:

(1) DESSEDG is a combination of EDM and mechanical grinding, the introduction of EDM make material removal rate increased by 2 to 4 times than simply mechanical grinding.

(2) Since sheet electrode is very thin $(0.05 \sim 0.15 \mathrm{~mm})$ and is sent very close to the surface of non-conducting engineering ceramics, it can be considered that the spark discharge energy between the sheet electrode and the grinding wheel directly acts on the surface of the non-conductive workpiece. The discharge process is not affected by mechanical properties of processed materials, and can be stable and uniform in processing area. Mechanical grinding is mainly used for removal of EDM metamorphic layer, the mechanical grinding force after EDM can be much more smaller than simply grinding, even the grinding wheel can be made by pure soft steel and grind with zero grinding force.

(3) Rough, medium and finishing processing of non-conductive engineering ceramics can be implemented on the same DESSEDG machine by adjusting the spark discharge and mechanical 
grinding parameters. For example, when rough machining large peak current, pulse width and grinding force are used, EDM plays a major role in this point; when finishing the small parameters are selected, the machining is dominated by mechanical grinding.

(4) DESSEDG uses non-conducting emulsion as working fluid, and doesn't need electrolyte in the electrochemical reaction to generate the discharge conditions. This can avoid the electrolysis polluting the environment, and corroding the machine tool.

(5) Spark discharge between sheet electrode and grinding wheel can dress the grinding wheel, and maintain its shape and accuracy.

\section{Processing Experiment}

In order to verify possibility of DESSEDG method, many experiments have been made with the experimental equipment.

Using a conductive grinding wheel made by 45\# steel, and copper sheet electrode of $25 \mathrm{~mm}$ wide and $0.15 \mathrm{~mm}$ thick, an non-conductive $\mathrm{Al}_{2} \mathrm{O}_{3}$ ceramic workpiece was machined. A groove of $25 \mathrm{~mm}$ wide and $1.5 \mathrm{~mm}$ deep has been made in the surface, as shows in Fig. 3. ${ }^{[5]}$ The material of the wheel is relatively soft, so the grinding effect was very weak; the spark discharge energy played a major role in the material removal. So, the material remove rate is very high, and the surface roughness is very poor.

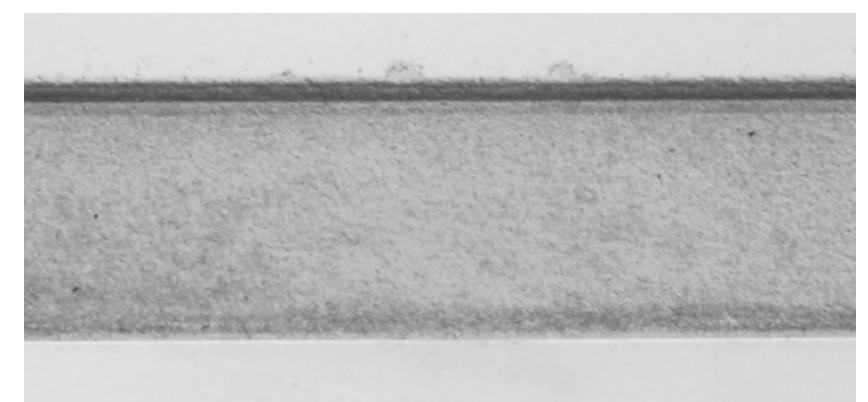

Fig. $3 \mathrm{Al}_{2} \mathrm{O}_{3}$ material sample machined by DESSEDG

\section{Conclusions}

DESSEDG is a very new method for non-conductive engineering ceramics. It has many advantages other methods do not have, and provides an effective solution for the problem of non-conductive engineering ceramics machining. So it has great research value and market prospects.

\section{Acknowledgements}

The authors gratefully acknowledge the financially support by the fund for science and technology research (01112) of the Ministry of Education, PRC.

\section{References}

[1] MOHRI N, FUKUZAWA Y, TANI T, et al: CIRP Annals - Manufacturing Technology Vol. 51(2002), p. 161-4

[2] KONIG W, DAUW D F, LEVY G, et al: Annals of the CIRP Vol. 37(1998), p. 623-31.

[3] Liu Jinchun, Zhao Jiaqi: Non-Conventional Machining, Machinery Industry Press, China Beijing (1993), In Chinese

[4] Li Minghui: Theoretical Basis of EDM, National Defense Industry Press, China Beijing (1989), In Chinese

[5] Y.H. Liu, X.P. Li, et al: Journal of Materials Processing Technology Vol. 208 (2008), p. 245-250 